\title{
THE AMALGAMATION PROPERTY FOR VARIETIES OF LATTICES
}

\author{
BY
}

\author{
ALAN DAY AND JAROSLAV JEŽEK ${ }^{1}$
}

\begin{abstract}
There are precisely three varieties of lattices that satisfy the amalgamation property: trivial lattices, distributive lattices, and all lattices.
\end{abstract}

1. Introduction. In [5], Jónsson showed that the variety of all lattices satisfied the (strong) amalgamation property and in [7] Pierce proved the similar (weak) version for distributive lattices. Grätzer, Jónsson and Lakser supplied the first negative results in $[3]$ by showing that the only varieties of modular lattices that satisfied the amalgamation property were varieties of distributive lattices (there are two such varieties, $\tau=\mathcal{L}(x=y)$ and $D)$. This result is crucial in that it forces $N_{5}$, the pentagon, into any nondistributive $\mathcal{V}$ satisfying (AP). Using this fact and the description of primitive lattices from Ježek and Slavík [4], Slavík, [8], showed that such a nondistributive variety, $\mathcal{V}$, satisfying (AP) must contain all primitive lattices. In this paper we complete the process started by Slavík, though by slightly different methods, and show that $\mathcal{V}=\mathcal{L}$. $^{1}$

Slavik's approach involved ingenious arguments using his notion of $A$-decomposability. This notion defines when a lattice, $L$, has to be the amalgamation of two proper sublattices, $S_{1}$ and $S_{2}$, thus providing an inductive procedure to force larger lattices into $\mathcal{V}$. Our first result is a complete characterization of this important idea. Slavík then used $A$-decomposability on the construction procedures for primitive lattices to produce his results. We apply it to $B$, the class of so-called bounded lattices introduced by McKenzie in [6]. By Day $[1]$, we have that $\operatorname{HSP}(B)=\mathcal{L}$ and by Day $[2]$, all members of $B$ are generated by the interval construction from the lattice, 1. Our second result implies that for $L \in B$, if $L \in \mathcal{V}$ then $L[I] \in \mathcal{V}$, and this completes the proof that $\mathcal{V}=\mathcal{L}$.

The authors are indebted to V. Slavík and J. B. Nation for stimulating discussions on this problem.

2. A-decomposibility. In this section we characterize Slavík's important notion of:

(2.1) Definition [8]. Let $L$ be a finite lattice and let $S_{1}$ and $S_{2}$ be proper sublattices of $L . L$ is called $A$-decomposable by means of $S_{1}$ and $S_{2}$ [Notation: $\left.L=A\left(S_{1}, S_{2}\right)\right]$ if $L=S_{1} \cup S_{2}$ and for any lattice, $Z$, and lattice monomorphisms $f_{i}: S_{i} \nrightarrow Z$ with $f_{1} \mid S_{1} \cap S_{2}=f_{2} \uparrow S_{1} \cap S_{2}, f=f_{1} \cup f_{2}$ is a lattice monomorphism from $L$ into $Z$.

Since the variety of all lattices satisfies (AP) by [5], the free amalgamation of $S_{1} \cap S_{2} \hookrightarrow S_{i}, i=1,2$, always exists. If $L=A\left(S_{1}, S_{2}\right)$ then $L$ is this

Received by the editors April 15, 1983.

1980 Mathematics Subject Classification. Primary 06B05; Secondary 06B20, 08B25.

${ }^{1}$ This research was supported by NSERC Operating Grant A-8190. 
free amalgamation in $\mathcal{L}$. Moreover if $\mathcal{V}$ satisfies (AP) and $S_{1}, S_{2} \in \mathcal{V}$, we obtain $L=A\left(S_{1}, S_{2}\right) \in \mathcal{V}$.

Given lattices $S_{1}$ and $S_{2}$ with $S=S_{1} \cap S_{2}$, there are three relatively simple ways to amalgamate the diagram

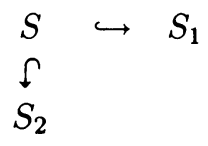

The most familiar method is, by [5], using the McNeil Completion of $\left(S_{1} \cup S_{2}\right.$, ᄃ $)$ where $x \sqsubseteq y$ in $S_{1} \cup S_{2}$ iff there exists a $z \in S$ with $x \leq z$ in $S_{i}$ and $z \leq y$ in $S_{j}$ for some $i, j \in\{0,1\}$. Since $\operatorname{MC}\left(S_{1} \cup S_{2}\right.$, $)$ preserves existing joins and meets, there are lattice monomorphisms $f_{i}: S_{i} \longmapsto \mathrm{MC}\left(S_{1} \cup S_{2}\right.$, ᄃ) with $f_{1}\left|S=f_{2}\right| S$. This provides us with our first necessary condition for $L=A\left(S_{1}, S_{2}\right)$.

(2.2) LEMMA. If $L=A\left(S_{1}, S_{2}\right)$, then $S_{1}$ and $S_{2}$ satisfy $O\left(S_{1}, S_{2}\right): x \leq y, x \in S_{i}$ and $y \in S_{j}$ imply there exists $z \in S$ with $x \leq z$ and $z \leq y$.

The McNeil Completion is not the only way in which $\left(S_{1} \cup S_{2}\right.$, ᄃ) can be completed. Let $\operatorname{IC}\left(S_{1}, S_{2}\right)$ be the set of all $\left(S_{1}, S_{2}\right)$-ideals. That is: $I \in \operatorname{IC}\left(S_{1}, S_{2}\right)$ if (1) $x \in I$ and $y \sqsubseteq x$ imply $y \in I$ and (2) $x, y \in I \cap S_{i}$ imply $x \vee_{i} y \in I$. Clearly the intersection of $\left(S_{1}, S_{2}\right)$-ideals is again such and therefore $\operatorname{IC}\left(S_{1}, S_{2}\right)$ is indeed a lattice. It is easy to check that $f_{i}: S_{i} \rightarrow \operatorname{IC}\left(S_{1}, S_{2}\right)$ by $f_{i}(x)=\downarrow x=\left\{y \in S_{1} \cup S_{2}\right.$ : $y \sqsubseteq x\}, i=1,2$, are lattice monomorphisms with $f_{1}\left|S=f_{2}\right| S$. Dually we can define the $\left(S_{1}, S_{2}\right)$-filter completion, $\mathrm{FC}\left(S_{1}, S_{2}\right)$.

We need to describe certain joins and meets of $\operatorname{IC}\left(S_{1}, S_{2}\right)$ in the special case where $L=S_{1} \cup S_{2}$ for sublattices $S_{1}$ and $S_{2}$ satisfying $O\left(S_{1}, S_{2}\right)$. Clearly $\downarrow x \cap \downarrow y=$ $\downarrow x \wedge y$ for all $x, y \in L$. In order to calculate $\downarrow x \vee \downarrow y$ we need a technical lemma.

(2.3) Lemma. For $x \in S_{1} \backslash S_{2}$ and $y \in S_{2} \backslash S_{1}$, there exists $x^{\prime} \in S_{1}$ and $y^{\prime} \in S_{2}$ such that $\downarrow x \vee \downarrow y=\downarrow x^{\prime} \vee \downarrow y^{\prime}$ and there exists $z \in S$ with $z \leq x^{\prime} \wedge y^{\prime}$.

PRoOF. Take $x \in S_{1} \backslash S_{2}$ and $y \in S_{2} \backslash S_{1}$. Since $x \wedge y \in L$ we have $x \wedge y \in S_{1}$ or $x \wedge y \in S_{2}$. Without loss of generality assume $x \wedge y \in S_{1}$. By $O\left(S_{1}, S_{2}\right)$, there exists $u \in S$ with $x \wedge y \leq u \leq y$ and by definition $x^{\prime}=x \vee u \in \downarrow x \vee \downarrow y$. Now $x^{\prime} \in S_{1}$ and by defining $y^{\prime}=y$ we get $\downarrow x \vee \downarrow y=\downarrow x^{\prime} \vee \downarrow y^{\prime}$.

(2.4) Definition. For $i \in\{1,2\}$ and $S_{i}^{\prime}=S_{i} \cup\{0,1\}$ define $\alpha_{i}: L \rightarrow S_{i}^{\prime}$ by $\alpha_{i}(x)=\bigvee\left\{u \in S_{i}^{\prime}: u \leq x\right\}$.

Note that for $x \in S_{1} \backslash S_{2}$ and $y \in S_{2} \backslash S_{1}$ we have:

(1) $x \wedge y \leq \alpha_{2}(x)<x$ or $x \wedge y \leq \alpha_{1}(y)<y$,

(2) $\alpha_{2}(x)<z \leq x$ implies $z \in S_{1} \backslash S_{2}$,

(3) $\alpha_{2}(x) \in\{0,1\} \cup S$ (by 2.2).

(2.5) Lemma. For $x \in S_{1} \backslash S_{2}$ and $y \in S_{2} \backslash S_{1}$ define $x_{0}=x, y_{0}=y$ and $x_{n+1}=x_{n} \vee \alpha_{1}\left(y_{n}\right), y_{n+1}=y_{n} \vee \alpha_{2}\left(x_{n}\right)$. Then $\downarrow x \vee \downarrow y=\bigcup\left\{\downarrow x_{n} \cup \downarrow y_{n}: n \geq 0\right\}$.

Proof. Easy induction gives $x_{n} \in S_{1}$ and $y_{n} \in S_{2}$ for each $n$ as well as $x_{n}, y_{n} \in \downarrow x \vee \downarrow y$. However the union is clearly an $\left(S_{1}, S_{2}\right)$-ideal.

If $a$ is covered by $b$ in $L$ we call $a$ (resp. $b$ ) a lower (resp. upper) neighbour of $b$ (resp. $a$ ). We let $\operatorname{LN}(a)$ (resp. $\mathrm{UN}(a)$ ) be the set of all lower (resp. upper) neighbours of $a$. 
(2.6) THEOREM. Let $L$ be a finite lattice with $L=S_{1} \cup S_{2}$ for proper sublattices $S_{1}$ and $S_{2}$ and define $S=S_{1} \cap S_{2} . L$ is A-decomposable by means of $S_{1}$ and $S_{2}$ if and only if $S_{1}$ and $S_{2}$ also satisfy:

$O\left(S_{1}, S_{2}\right): x \in S_{i}, y \in S_{j}$ and $x \leq y$ imply the existence of $z \in S$ with $x \leq z$ and $z \leq y$.

$\mathrm{LN}\left(S_{1}, S_{2}\right):$ For all $x \in S, \mathrm{LN}(x) \subseteq S_{1}$ or $\mathrm{LN}(x) \subseteq S_{2}$.

$\mathrm{UN}\left(S_{1}, S_{2}\right):$ For all $x \in S, \mathrm{UN}(x) \subseteq S_{1}$ or $\mathrm{UN}(x) \subseteq S_{2}$.

Proof. Assume firstly that $L=A\left(S_{1}, S_{2}\right)$. By Lemma (2.3), $O\left(S_{1}, S_{2}\right)$ holds and by definition the map $x m \downarrow x$ of $L$ into $\operatorname{IC}\left(S_{1}, S_{2}\right)$ must be a lattice monomorphism. If there existed an element $u \in S$ with $x \in \operatorname{LN}(u) \backslash S_{2}$ and $y \in \operatorname{LN}(u) \backslash S_{1}$, $\downarrow x \cup \downarrow y$ would be an $\left(S_{1}, S_{2}\right)$-ideal (observe that $\left.S_{2} \cap(\downarrow x \cup \downarrow y) \subseteq \downarrow y\right)$ and therefore $\downarrow x \vee \downarrow y \neq \downarrow(x \vee y)$. Therefore $\operatorname{LN}\left(S_{1}, S_{2}\right)$ and dually $\mathrm{UN}\left(S_{1}, S_{2}\right)$ hold.

Conversely assume the three conditions hold and take lattice monomorphisms $f_{i}: S_{i} \mapsto Z$ with $f_{1} \uparrow S=f_{2} \uparrow S$. We must show $g=f_{1} \cup f_{2}$ is a monomorphism.

Claim 1. $x<y$ implies $g(x)<g(y)$.

Assume $x \in S_{1} \backslash S_{2}$ and $y \in S_{2} \backslash S_{1}$. By $O\left(S_{1}, S_{2}\right)$ there is a $z \in S$ with $x \leq z \leq y$. Moreover one of these inequalities must be strict. Therefore $g(x) \leq g(z) \leq g(y)$ with one of these inequalities strict, hence $g(x)<g(y)$.

Claim 2. $g(x \vee y)=g(x) \vee g(y)$.

We need only consider the case where $x \in S_{1} \backslash S_{2}$ and $y \in S_{2} \backslash S_{1}$. By easy induction we obtain $g(x) \vee g(y)=g\left(x_{n}\right) \vee g\left(y_{n}\right)$ for all $n$.

Now if $x_{n}, y_{n}<x \vee y$ for all $n$, there exists $k$ with $x_{k+1}=x_{k} \in S_{1} \backslash S_{2}$ and $y_{k+1}=y_{k} \in S_{2} \backslash S_{1}$. Therefore $\alpha_{1}\left(y_{k}\right) \leq x_{k}$ and $\alpha_{2}\left(x_{k}\right) \leq y_{k}$. By 2.4(1) we get $x_{k} \wedge y_{k} \in\left\{\alpha_{1}\left(y_{k}\right), \alpha_{2}\left(x_{k}\right)\right\}$. Hence $x_{k} \wedge y_{k} \in S$ by 2.4(3). Now 2.4(2) supplies the contradiction to $\mathrm{UN}\left(S_{1}, S_{2}\right)$. Therefore for some $n, x_{n}=x \vee y \geq y_{n}$ and $g(x) \vee g(y)=g(x \vee y)$.

Claim 3. $g(x \wedge y)=g(x) \wedge g(y)$.

By duality.

Therefore $g: L \rightarrow Z$ is indeed a lattice monomorphism and $L=A\left(S_{1}, S_{2}\right)$.

The above characterization makes it trivial to obtain certain properties of $A$ decomposable lattices.

(2.7) Corollary. If $L=A\left(S_{1}, S_{2}\right)$ and $S_{i} \leq T_{i}<L, i=1,2$, then $L=$ $A\left(T_{1}, T_{2}\right)$.

(2.8) COROLlaRY. If there exists $0<a \leq b<1$ in $L$ with $L=\uparrow a \cup \downarrow b$, then $L=A(\uparrow a, \downarrow b)$.

3. $\mathcal{V}=\mathcal{L}$. Let $\mathcal{V}$ be a nondistributive variety of lattices satisfying the amalgamation property. By $[3]$ we have $N_{5} \in \mathcal{V}$. We wish to show $\mathcal{V}=\mathcal{L}$.

(3.1) LEMMA. Let $L$ be a finite lattice with $I=[u, v] \leq L$. If there exists $\theta \in \operatorname{Con}(L)$ with $I=[I] \theta$, then $L[I]$ is a sublattice of a product of $L$ and $L / \theta[I / \theta]$.

Proof Let $\psi=\operatorname{Ker} f$ for the canonical $f: L[I] \rightarrow L$ and define $\bar{\theta} \in \operatorname{Con}(L[I])$ by $x \bar{\theta} y$ iff $x, y \in L \backslash I$ and $x \theta y$ or $x, y \in I \times\{i\}$ and $f(x) \theta f(y)$ for $i \in 2$. Easy calculations show that $\bar{\theta} \in \operatorname{Con}(L[I]), L[I] / \bar{\theta} \leq(L / \theta)[u / \theta, v / \theta]$ and $\psi \wedge \bar{\theta}=\Delta_{L[I]}$. 
(3.2) COROllaRY. If $L$ is semidistributive, $0 \prec u \leq v \prec 1$, and $I=[u, v]$, then $L[I]$ is a sublattice of a product of $L$ and $N_{5}$.

ProOF. Let $\kappa(u)=\bigvee\{x \in L: x \wedge u=0\}$ and $\lambda(v)=\bigwedge\{y \in L: y \vee v=1\}$. Then we have a homomorphism $f: L \rightarrow \mathbf{2}^{2}$ with congruence classes $[u, v],[\lambda(v), \kappa(u)]$, $[0, v \wedge \kappa(u)]$ and $[u \vee \lambda(v), 1]$.

We would have liked a direct proof that $L \in B \cap \mathcal{V}$ implies $L[I] \in B \cap \mathcal{V}$ but this seems impossible. The following variation, however, does do the job.

(3.3) Lemma. For $L \in B \cap \mathcal{V}$ and $I=[u, v] \leq L, i \in \mathbf{2}$, then $(L \times \mathbf{2})[(u, i),(v, i)]$ $\in \mathcal{V}($ and $B)$.

Proof. By induction on $|L|$. Assume $i=1$. If $v=1$, then $(L \times 2)[(u, 1),(v, 1)] \leq$ $L \times \mathbf{3} \in \mathcal{V}$. If $v<1$, there is a co-atom $m, v \leq m \prec 1$, and for $p=\lambda(m), L=$ $\downarrow m \cup \uparrow p$. Therefore $L \times \mathbf{2}$ can be pictured as in Figure (i). Since $J=[(0,1),(m, 1)]$ is a congruence class of the homomorphism $f: L \times \mathbf{2} \rightarrow \mathbf{2}^{2}$, we can double $J$ to produce a lattice that is a subdirect product of $L \times 2$ and $N_{5}$, hence a lattice in $\mathcal{V}$. The congruence classes modulo the homomorphism $g:(L \times 2)[J] \rightarrow N_{5}$ produce the diagram in Figure (ii). Again since $B_{0}$ is a congruence class of this lattice, we can double this interval to produce a lattice $M \in \mathcal{V}$ as in Figure (iii). Now let $J$ be the interval I considered as lying in the congruence class labelled $B$ in Figure (iii), and consider the lattice $M[J]=A \cup B_{0} \cup B_{1} \cup C \cup D \cup B[J]$. By defining $S_{1}=A \cup B_{0} \cup B_{1} \cup C \cup D$ and $S_{2}=B_{0} \cup B_{1} \cup B[J]$, we obtain $M[J]=A\left(S_{1}, S_{2}\right)$. Since $S_{1}$ is the lattice of Figure (ii), $S_{1} \in \mathcal{V}$. Since $S_{2}=$ $A\left(B_{0} \cup B[J], B_{1} \cup B[J]\right), S_{2} \in \mathcal{V}$ if and only if these two lattices belong to $\mathcal{V}$. But $B=[0, m]$ with $|B|<|L|$, and these two lattices are $B \times 2$ with the interval $I \times\{i\}$ split upstairs and downstairs respectively. By induction then $S_{2} \in \mathcal{V}$ and hence $M[J] \in \mathcal{V}$. Since $(L \times \mathbf{2})[(u, 1),(v, 1)] \cong A \cup B[J] \cup C \cup D \leq M[J]$, this lattice is in $\mathcal{V}$.

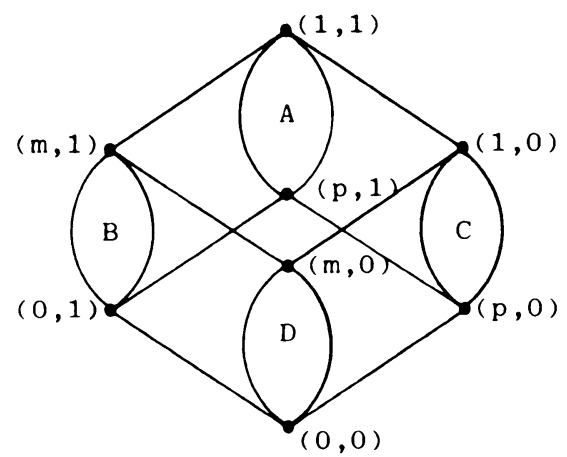

FIGURE (i) 

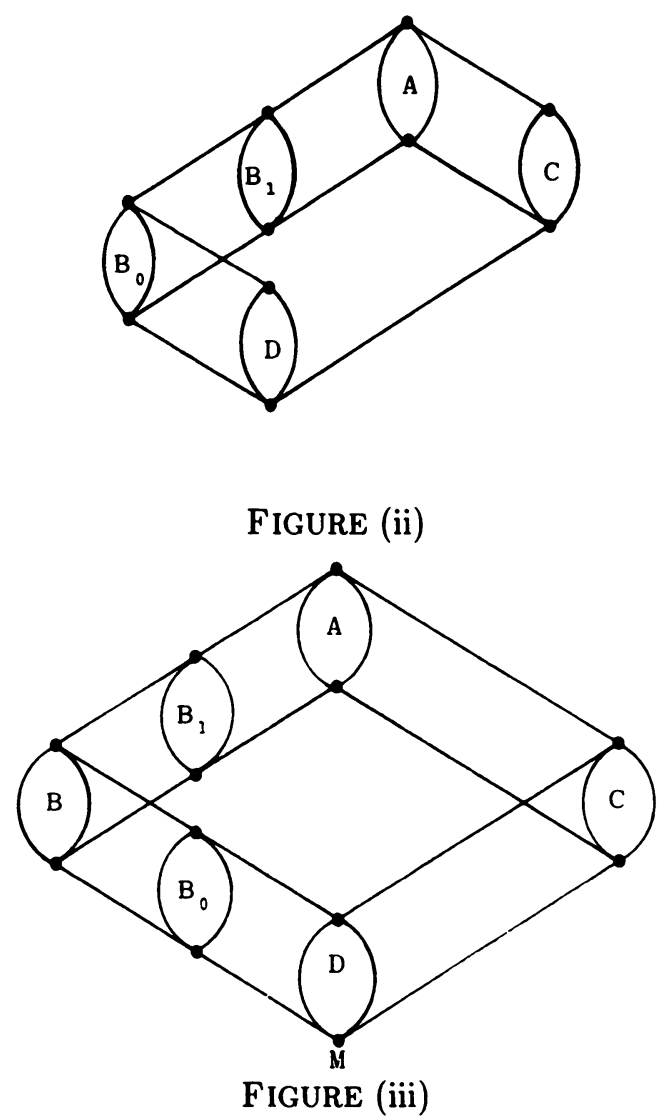

The proof for $i=0$ follows by symmetry.

(3.4) THEOREM. The only varieties of lattices that satisfy the amalgamation property are $\mathcal{T}, D$, and $\mathcal{L}$.

ProOF. If $\mathcal{V}$ is a nondistributive variety satisfying satisfying (AP), then by [3], $N_{5} \in \mathcal{V}$. Lemma (3.3) implies that for every $L \in B$, if $L \in \mathcal{V}$ then $L[I] \in \mathcal{V}$ since $L[I] \leq(L \times 2)[I \times\{i\}]$. By $[2], B \subseteq \mathcal{V}$ and by $[\mathbf{1}], \mathcal{L}=\mathbf{H S P}(B) \subseteq \mathcal{V}$.

Since our proof requires only that $N_{5} \in \mathcal{V}$ it would be of interest to have an elementary proof (as opposed to [3]) that if $\mathcal{V}$ satisfies (AP) and $M_{3} \in \mathcal{V}$ then $N_{5} \in \mathcal{V}$. Such a proof is not known to the authors.

(3.5) COROLLARY. $\mathcal{L}$ is the only variety of lattices satisfying the strong amalgamation property.

ProOF. D does not satisfy (SAP). Whether or not $T$ has the strong amalgamation property depends directly on whether or not the empty lattice, $\phi$, is allowed. If $\phi \in T$, then

$$
\begin{aligned}
& \phi \\
& \mathfrak{f} \\
& \{y\}
\end{aligned} \quad \hookrightarrow\{x\}
$$

has no strong amalgamation in $T$. 


\section{REFERENCES}

1. A. Day, Splitting lattices generate all lattices, Algebra Universalis 7 (1977), 163-169.

2. Characterizations of finite lattices that are bounded-homomorphic images or sublattices of free lattices, Canad. J. Math. 31 (1979), 69-78.

3. G. Grätzer, B. Jónsson and H. Lakser, The amalgamation property in equational classes of modular lattices, Pacific J. Math. 45 (1973), 507-524.

4. J. Ježek and V. Slavík, Primitive lattices, Czechoslovak Math. J. 29 (1979), 595-634.

5. B. Jónsson, Universal relational systems, Math. Scand. 4 (1956), 193-208.

6. R. McKenzie, Equational bases and nonmodular lattice varieties, Trans. Amer. Math. Soc. 174 (1972), 1-43.

7. R. S. Pierce, Introduction to the theory of abstract algebras, Holt, Rinehart and Winston, New York, 1968.

8. V. Slavik, $A$ note on the amalgamation property in lattice varieties, Colloq. Math. Soc. Janos Bolyai (Szeged) (to appear).

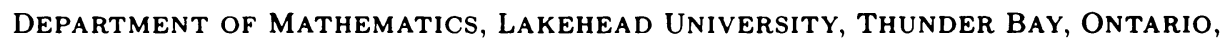
CANADA P7B 5E1

Department of MATHEMATICS, ChaRles University, MFF, SOKOlovská 83, 18600 Praha 8, Czechoslovakia 